\title{
Dental Operating Microscope in Endodontics-A Review
}

\author{
Prof. (Dr.) Utpal Kumar Das ${ }^{1}$, Dr. Subhasis Das ${ }^{2}$ \\ ${ }^{1}$ (HOD,Department of Conservative Dentistry \& Endodontics, Guru Nanak institute of Dental Science \& \\ Research, Kolkata,India) \\ 2(PGT,Department of Conservative Dentistry \& Endodontics, Guru Nanak institute of Dental Science \& \\ Research, Kolkata,India)
}

\begin{abstract}
Traditional endodontics has been based on feel not sight. Together with radiographs and electronic apex locators this blind approach has produced surprising success. There is, however, a significant failure rate, especially in long-term. Magnification helps the user not only to see more, but to see well. High levels of magnification increase the aggregate amount of visual information available to endodontists for diagnosing and treating dental pathology. Initially resisted, there has been a recent surge of interest in microscope enhanced dentistry among endodontists. Despite their higher price tags, however, when the dental operating microscope is fully integrated into an endodontic practice and used to its fullest potential, a return on investment is improved ergonomics and zero defect endodontics.

Keywords:Dental Operating Microscope (DOM), Endodontics, Ergonomics, Magnification.
\end{abstract}

\section{Introduction}

"You can only treat what you can see! " Prof. Syngcuk Kim.

In 1978 Dr. Apotheker and Dr. Jako brought the concept of extreme magnification, in the form of an operating microscope, into dentistry. They postulated that the tremendous improvements in visual acuity, made possible through the use of the operating microscope, would be beneficial to the discipline of endodontics[1].

The first commercially available Dental Operating Microscope (DOM) named Dentiscope(Fig.1) introduced in 1981 by Chayes-Virginia Inc, (Evansville, IN ) was poorly configured and ergonomically difficult to use. It was capable of only one magnification (8x), was positioned on a floor stand and poorly balanced, had only straight binoculars, and had a fixed focal length of $250 \mathrm{~mm}$, used angled illumination instead of confocal illumination did not gain wide acceptance, and the manufacturer ceased manufacturing it shortly after its introduction (1986). Its market failure was more a function of its poor ergonomic design than its good optical properties [2].

In the late 1980s, San Diego endodontist Dr. Gary Carr concluded that the incredible magnification and illumination made possible with the microscope could be beneficial to endodontists and started promoting the usage of the DOM as a crucial piece of the armamentarium used in the improvement of outcomes of endodontic apical surgeries. Howard S Selden [1986] was the first endodontist to publish an article on the use of the DOM in endodontics where he discussed its use in the conventional treatment of a tooth, not in surgical endodontics.

In 1995, the American Association of Endodontists (AAE) formally recommended to the Commission on Dental Accreditation (CODA) of the American Dental Association (ADA) that microscopy training be included in the new Accreditation Standards for Advanced Specialty Education Programs in Endodontics. At the commission's meeting in January 1996, the proposal was agreed on, and in January 1997, the new standards, making microscopy training mandatory, became effective [3].

In 1999, Gary Carr, introduced a DOM that had Galilean optics and that was ergonomically configured for dentistry, with several advantages that allowed for easy use of the scope for nearly all endodontic and restorative procedures[4]. It used a confocal illumination module so that the light path was in the same optical path as the visual path, gave far superior illumination than the angled light path of the earlier scope, gained rapid acceptance within the endodontic community, and is now the instrument of choice not only for endodontics but for periodontics and restorative dentistry as well (Fig. 2) [2].

\section{Advantages of Dental Operating Microscope}

There are five basic advantages in using the DOM and accompanying documentation systems (digital microphotography and videography) for an endodontic specialist include: increased visualization, improved Quality and precision of treatment, enhanced ergonomics, ease of proper digital documentation and increased communication ability through integrated video[5]. 


\subsection{Increased Visualization}

Carr reported that the human eye, when unaided by magnification, has the inherent ability to resolve or distinguish two separate lines or entities that are at least 200 microns, or $0.2 \mathrm{~mm}$, apart [2]. If the lines are closer together, two separate entities or the objects will appear as one [6].

Most people cannot refocus at distances closer than 10 to $12 \mathrm{~cm}$. As the eye-subject distance (i.e. focal length) decreases, the eyes must converge, creating eyestrain. Furthermore, as one ages, the ability to focus at closer distances is compromised, caused by the lens of the eye losing flexibility with age (presbyopia). As the focal distance decreases, depth of field also decreases. Considering the problem of the uncomfortable proximity of the practitioner's face to the patient, moving closer to the patient is not a satisfactory solution for increasing a clinician's resolution.

Alternatively, a common DOM can raise the resolving limit from $0.2-0.006 \mathrm{~mm}$, thus with magnification the resolution of the human eye improves dramatically .In addition to having up to six levels of magnification ranging from $2 \mathrm{x}$ to $26 \mathrm{x}$ available, illumination is a critical component in increasing visualization.

Most microscopes are equipped with an integrated coaxial light source that allows for unobstructed, shadow-free illumination of the operating field which allows for significantly improved visualization of even the most difficult to access areas of the oral cavity $[4,7]$.

\subsection{Improved Quality and precision of treatment}

The visual information provided by the operating microscope is, in fact, not indicative of the magnification that is being employed. The actual amount of visual information is the area under the scope and is therefore the number of horizontal pixels multiplied by the number of vertical pixels. A microscope at $10 x$ magnification provides 25 times the information compared to that obtained through the use of entry-level loupes (2x) and over 10 times that of $3 \times$ power loupes (Fig.3). As magnification increases, the depth and diameter of the field-of-view of the operating field decrease. There is an increased demand at higher magnification for improved control of the micromotor muscles and joints (fingers and wrists) that can require stabilization of the gross motor joints (elbow and shoulder) with microendodontist' chairs[8].

Shanelec and Tibbets[1998] reported that clinician, working without magnification, can make movements that were $1-2 \mathrm{~mm}$ at a time. At $20 \times$ magnification, the refinement in movements can be as little as $10-20$ microns $(10-20 / 1000$ of a $\mathrm{mm})$ at a time. It is useful therefore to note that the limitation to precision of treatment is not in the hands but in the eyes [9].

Baldissara et al. [1998] showed that the experienced clinician with a sharp, new explorer can determine marginal gaps of around 36 microns with a tactile sense. Thus, it can be assumed that when magnification is greater than $6 \times$ powers, the reliance on an explorer and tactile means of inspection significantly decreases [10].

The precision of treatment studies by Leknius and Geissberger, [1995] as well as by Zaugg et al. [2004] demonstrated that as magnification is incorporated, procedural errors decrease significantly. In the latter study, the inclusion of a microscope resulted in fewer errors than when a set of loupes was used [11, 12].

\subsection{Improved \& Ideal treatment Ergonomics}

With DOM improved ergonomics is realized on many levels, the most obvious being improved posture. With microscope, the clinician is able to practice while looking straight ahead without having to either bend forward in an effort to see better (causing lower back pain), or raise the patient horizontally in order to bring the oral cavity closer to the clinician(causing neck pain). The microscope allows for $100 \%$ of the retina to be focused on the site [8].

By operating in a more upright, neutral and balanced posture, the endodontist is less likely to experience strain, tension or fatigue of neck of lower back muscles which allows one to focus completely on the task at hand and able to work comfortably for extended periods of time. This can enable the endodontist to provide more dentistry in fewer visits, increasing productivity and making more happy patients(Fig.4). Ergonomics is also improved during digital documentation because intra-operative images can be captured very efficiently by the assistant without interrupting treatment [13, 14, 15].

Moreover microscopes with a long working distance allow endodontist and his team members, distance from the patient during dental work reducing the risk of exposure to aerosols and spatter thus without putting at risk their own health and physical condition[16, 17, 18].

\subsection{Ease of Proper Digital Documentation Capabilities}

With the optional addition of a beam-splitting device, one is able to capture digital photos and record real-time video at multiple magnifications, by integrating various types of digital recording devices, such as an SLR and/or video camera and saved in hard drives and mini DV tapes or directly to DVD (Fig.5) [8]. 
Digital documentation capabilities enable the clinician to efficiently capture and share with patients what is seen during an examination pre-operatively, intra-operatively and post-operatively and stored in patients chart. This is especially useful when unforeseen problems are encountered. This can leads to greater rates of case acceptance, increasing patient's level of trust and confidence and significantly streamline the amount of time required in gaining it $[19,20]$.

The usage of documentation for medico-legal, insurance, patient communication, and lecturing purposes, as well as for communication with staff or colleagues, is also impressive [21, 22].

\subsection{Increased Ability to Communicate through Integrated Video}

Mehrabian has shown that as much as $55 \%$ of the understanding that occurs in verbal communication is through visual cues, and only $7 \%$ of the comprehension comes from the words we use. Stated differently, patients remember more of what they see, and what they see is what they hear [23].

Adding video to the microscope have found useful in providing information both to patients and to auxiliaries, as the microscope, like an intraoral camera, allows them co-observation of the multiple steps during the procedure in real time but also become involved in a particular portion of the procedure (Fig.6).

Finally, the ability to quickly edit video files and integrate them into programs, such as Windows Movie Maker and PowerPoint and the live video stream opens up tremendous abilities to share information and discussions with colleagues, either in a lecture format, where live video can be transferred from the scope to an LCD projector and transmitted onto a screen for the audience to see, or be captured on tape or hard drive and shared with colleagues at high magnification allowing greater learning experience.

Nowadays, the Internet has opened up the possibility of watching streaming live procedures, documented through the microscope, and a whole new level of continuing education has emerged, as lectures and procedures become viewable via computer from the comfort of one's own home [8].

\section{Disadvantages}

There are some disadvantages, especially at the initial stages, most important one is the need for specific training: as a DOM has a restricted working field, $11 \mathrm{~mm}-55 \mathrm{~mm}$.An operator using a DOM can see only the tip of the instruments, and they are used in delicate movements of small amplitude. Other disadvantages include the relatively high initial cost of the equipment and instruments, the need for retraining of the auxiliary staffs, and an adjustment period for the new treatment paradigms and operator postures, may increase treatment costs and reduce initial productivity, besides the need for rescheduling [24].

\section{Position Statement of AAE on Use of Microscopes and Other Magnification Techniques}

Position Statement of AAE on Use of Microscopes and Other Magnification Techniques, published in 2012 recommends the following procedures in Endodontics can be benefitted from the use of the microscope [25]:

- locating hidden canals that have been obstructed by calcifications and reduced in size;

- removing materials such as solid obturation materials (silver points and carrier-based materials), posts or separated instruments;

- removing canal obstructions;

- assisting in access preparation to avoid unnecessary destruction of mineralized tissue,

- repairing biological and iatrogenic perforations;

- locating cracks and fractures that are neither visible to the naked eye nor palpable with an endodontic explorer;

- facilitating all aspects of endodontic surgery, particularly in root-end resection and placement of retrofilling material.

\section{Use of Dental Operating Microscope in endodontic therapy}

In all areas, from exposure of the access cavity and preparation to three-dimensional obturation and post endodontic management, the microscope provides major advantages over working without appropriate magnification. As a result, the use of the microscope can be expressly recommended for the following specific indications and special aspects:

\subsection{Examination, diagnosis, and treatment planning}

With enhanced visualization, the clinician's ability to diagnose problems in the earlier stages of a disease process is possible. High-powered magnification allows endodontists to identify a microscopic blemish, colour alteration, tiny amounts of plaque collecting within the grooves, microscopic amounts of chalky white demineralization around the grooves, and tiny amounts of flaking of darkened carious tooth structure within the 
crevices of these grooves. Treatments also can be performed with a greater level of precision, thereby reducing the occurrence of failures and the need of redos [20, 26, 27].

\subsection{Diagnosis of cracked teeth}

Microfractures and longitudinal fractures which are often difficult to diagnose clinically and represent signs of occlusal damages which include cracks in teeth or restorations, craze lines, wear facets, cracks at slightly elevated marginal ridges, or areas where the enamel has been worn by opposing porcelain, exposing dentin and thus causing sensitivity and pain can be viewed more precisely with DOM (Fig.7, 8) [28, 29].

\subsection{Better visualization of pulp chamber, canal orifices}

Magnification allows endodontists to better identify anatomical landmarks, within the pulp chamber-including the sides, overhanging remnants of the pulp chamber roof, initial perforations into the pulp, dentinal map, canal orifices and to differentiate between the pulp horns and the main body of pulp within the chamber [30].

\subsection{During instrumentation}

During instrumentation, the improved ability to see specific canals allows endodontists to maneuver files into canal openings with greater efficiency, to distinguish between vital and necrotic canals, and to detect tiny amounts of purulence or blood draining through specific canals or see any tiny amounts of necrotic pulp material that were not removed during canal instrumentation. So it is possible for an endodontist to determine if all canals are accessed and instrumented properly when a direct view might be difficult without removing excessive amounts of coronal tooth structure $[30,31]$.

\subsection{Locating hidden canals/canal systems}

Anatomical variations are not as rare or exotic as is frequently assumed as described by Walter Hess as early as 1917. Many of these important structures cannot be readily detected or treated with traditional endodontic treatment methods. If the radiographic image is examined more closely, there are often signs of unusual root and/or canal shapes like those caused by changes in the course of canal anatomy or root surface. An off center exposure or three dimensional image can provide further valuable information. Three rooted premolars, for example, are encountered in $6 \%$ of all first maxillary premolars. However, anatomical variations also include other complex structures like middle mesial canal (Fig. 9) in mandibular first molar and C-shaped canals in mandibular second molar $(7.6 \%)[32,33]$.

Without a doubt, the second mesiobuccal canal in maxillary molars (16\% and $78 \%$ in vivo), which is often difficult to localize and prepare, is the reason why the failure rate is highest in first maxillary molars (Fig. 10). Virtually all studies point to distinct advantages in the localization and treatment of this highly complex anatomical variation when using a dental microscope.

The introduction of the dental microscope and the associated ability to inspect the root canals - both orthograde and retrograde - have fundamentally changed our understanding of dental morphology and its complexity $[27,34]$.

\subsection{Identification and removing of Obliterations and calcifications}

These signs occur to a greater or lesser extent in $50 \%$ of all teeth, impairing instrumentation considerably or essentially preventing treatment of the canal system (Fig. 11).

\subsection{Identification and removal of Denticles}

This specific form of calcification is also encountered very frequently, can block the canal entrance or even obstruct further instrumentation. Denticles can be found and negotiate readily with the help of a DOM (Fig. 12) [27].

\subsection{In Open apex cases}

Modern apexification therapies call for special treatment techniques and materials, the manipulation of which is facilitated significantly under a dental microscope (Figs. 13-14).

\subsection{Perforation repair}

Treatment of iatrogenic problem such as pulpal floor perforation, lateral root perforation and prognosis chiefly involve visualization of the problem so the microscope certainly plays a major role in this context (Fig. 15). 


\subsection{Removal of fractured post and instruments}

The enhanced vision with magnification and illumination from a microscope allows endodontist to observe the most coronal aspects of fractured post and broken instruments and to remove them without any major loss of tooth structure and perforations, the prognosis for preservation of the tooth is quite good.(Fig. 16)[35, 36].

\subsection{Microsurgical apicoectomy}

Incorporating microscopic approach in surgical endodontics, conceptualised by Prof. Kim in the 1990s with Use of the smaller retro mirrors it is possible to carefully examine the apical segment of the root end and perform an atraumatic, more moderated bevel apical resection procedures and permit a coaxial ultrasonic preparation into the root, better management of the bone structures thereby making minimally invasive class I retrograde cavity preparation and retrograde filling of the canal system and all its branches along the longitudinal axis of the root easy to perform [2,27].

Microsurgical flap design and Surgical soft-tissue management is also greatly enhanced by a microscopic approach, leading to faster healing, less traumatic soft-tissue management, and the advent of microsurgical suturing (7-0 to 10-0) techniques that minimize trauma and lead to rapid, primary intention wound healing and fewer post-operative pain and complications but also a much better prognosis than traditional procedures (Fig. 17) [2].

A study reported by Rubinstein and Kim [1999] confirmed healing in $96.8 \%$ of cases in the short term, and $91.5 \%$ in the long term follow-up is well beyond the success rates of conventional apicoectomy procedures. Another study points to an even greater discrepancy of $44.2 \%-60 \%$ for the traditional method and $91.1 \%$ for microsurgical techniques. [37, 38].

\subsection{Other uses}

There are others such as external cervical invasive resorption repairs, removing materials such as solid obturation materials (silver points and carrier-based materials), and other resorptive repairs that also benefit from a microscopic approach [2].

\section{Conclusion}

Exact therapy requires exact vision. High-quality endodontic therapy is the basis for long-term function and biologic success, ensuring that patients remain free of pain. State-of-the-art equipment and thorough clinical know-how are vitally important to reach this goal. Today, a small but growing number of clinicians are providing endodontic and endo-restorative treatments based on direct microscopic visualization of the pulp chamber and coronal portions of canal systems. This huge shift in clinical accuracy from low magnification "tactile-driven" endodontics to "vision-based" endodontics is bringing a revolution to the field of endodontics with greater success rate.

Microscope reduces distance and improves the overall treatment quality and encourages endodontists to review and perfect their own treatment concepts resulting in a positive impact on the entire practice structure but also increases the enjoyment of providing treatment.

\section{References}

[1]. Apotheker H. A Microscope for Use in Dentistry. J Microsurg. 1981; 3:7.

[2]. Gary B. Carr, Carlos A.F. Murgel-The Use of the Operating Microscope in Endodontics: Dent Clin N Am 54, 2010:191-214.

[3]. Selden HS. The dental-operating microscope and its slow acceptance. J Endod 2002; 28(3): 206-7.

[4]. CarrGB.Microscopes in Endodontics. J Calif Dent Assoc.1992: 20(11): $55-61$.

[5]. Use of the Dental Operating Microscope in Laser Dentistry: Seeing the Light Glenn A. van As-J Laser Dent 2007; $15(3): 122-129$.

[6]. Carr GB. Magnification and illumination in endodontics. In: Hardin JF, (Editor) Clark's Clinical Dentistry. (New York: Mosby, 1998) 4:1-14.

[7]. Behle C. Photography and the Operating Microscope in Dentistry. J Calif Dent Assoc. 2001; 29(10): 765771 .

[8]. Glenn A. van As,Extreme Magnification: Seeing the Light- www.ineedce.com

[9]. Tibbets LS, Shanelec DA. Periodontal Microsurgery. Dent Clin North Am. 1998; 42:339-359.

[10]. Baldissara P, Baldissara S, Scotti, R. Reliability of Tactile Perception Using Sharp and Dull Explorers in Marginal Opening Identification. Int J Prosth. 1998; 11(6):591-594.

[11]. Leknius C, Geissberger M. The Effect of Magnification on the Performance of Fixed Prosthodontic Procedures. J Calif Dent Assoc. 1995; 23(12):66-70.

[12]. Zaugg B, Stassinakis A, Hotz P. Influence of Magnification Tools on the Recognition of Simulated Preparation and Filling Errors. Schweiz Monatsschr Zahnmed. 2004; 114(9):890-896.

[13]. Chang BJ. Ergonomic benefits of surgical telescope systems: Selection guidelines. J Calif Dent Assoc. 2002; 30(2): 161-169.

[14]. Van As GA. Enhanced Acuity through Magnification: Clinical Application for Increased Visualization. Dentistry Today. 2001; 1(2): 40-42.

[15]. Friedman MJ, Landesman HM. Microscope Assisted Precision (MAP) Dentistry. J Calif dent Assoc. 1998 Dec; $26(12)$ : $900-5$. 
[16]. Cristian Comes, Anca Valceanu, Darian Rusu, Andreea Didilescu, Alexandru Bucur, Mirella Anghel, Veronica Argesanu, StefanIoan Stratul: A Study on the Ergonomical Working Modalities Using the Dental Operating Microscope (DOM). PART I: Ergonomic Principles in Dental Medicine; TMJ 2008, Vol. 58, No. 3 - 4

[17]. Cristian Comes, Anca Valceanu, Darian Rusu, Andreea Didilescu4, Alexandru Bucur, Mirella Anghel, Veronica Argesanu, StefanIoan Stratul; A study on the ergonomical working modalities using the dental operating microscope (DOM ). Part II: Ergonomic Design Elements of the Operating Microscopes. TMJ 2009, Vol. 59, No. 1

[18]. Andreea Didilescu, Cristian Comes, Darian Rusu, Mihai Bucur, Mirella Anghel, Veronica Argesanu, Stefan-Ioan Stratul; A study on the ergonomical working modalities using the dental operating microscope (DOM ). PART III: Ergonomical Features of Contemporary Top Dental Microscopes Commented; TMJ 2010, Vol. 60, No. 1.

[19]. MamtaTiwari,RajeshPodar:Benefits of the Dental Operating microscope: Terna J DentSci 2012;1:35-39.

[20]. The Dental Operating Microscope:The most valuable (and profitable) technology a dentist can own. Donato Napoletano, Inside Dentistry February 2010, Vol. 6, Issue 2.

[21]. Mora AF. Restorative Microdentistry: A New Standard for the $21^{\text {st }}$ Century. Prosthet Dent Rev. 1998; 1(3).

[22]. Van As GA. Digital Documentation and the Dental Operating Microscope. Oral Health. 91(12): 19-25.

[23]. Glenn A. van As:Digital Documentation and the Dental Operating Microscope: what you see is what you get: Int J Microdent 2009; $1: 30-41$.

[24]. Jose Roberto Moura Jr. Operating Microscopes in Restorative Dentistry: The Pursuit of Excellence, International Dentistry SA Vol. 10, No. 5

[25]. American Association of Endodontists: AAE Position Statement on Use of Microscopes and Other Magnification Techniques, www.aae.org, 2012.

[26]. Clark DJ, Sheets CG, Paquette JM. Definitive diagnosis of early enamel and dentin cracks based on microscopic evaluation. $J$ Esthet Restor Dent 2003; 15: 391-401.

[27]. Thomas Clauder:The Dental Microscope: An Indispensable Tool in Endodontic Practice, Reprint from "The Microscope in Dentistry: An Editorial Forum for Dental Professionals", published by Carl Zeiss Meditec AG, Jena, Germany.

[28]. Glenn A. van As: Evaluation of Enamel and Dentinal Cracks Using Methylene Blue Dye and the Operating Microscope, Inside Dentistry,July/August 2007, Volume 3, Issue 7.

[29]. Jack D Griffin, Jr: Efficient, Conservative Treatment of Symptomatic Cracked Teeth; Compendium / February 2006 Vol.27, No. 2.

[30]. John S. Mamoun ;A rationale for the use of high-powered magnification or microscopes in general dentistry; General Dentistry, January/February $2009 ;$ Pg. 18-26.

[31]. Frank J. Vertucci;Root canal morphology and its relationship to endodontic procedures; Endodontic Topics 2005,10, 3-29.

[32]. Jafarzadeh H., Wu Y.-N. : The C-shaped Root Canal Configuration: A Review. J Endod 2007; 33(5): $517-523$.

[33]. Seo M. S., Park D. S.: C-shaped root canals of mandibular second molars in a Korean population: clinical observation and in vitro analysis. Int Endod J, 2004; 37: 139-144.

[34]. Buhrley L J, Barrows MJ, Begole EA, Wenckus CS; Effect of magnification on locating the MB2 canal in maxillary molars. $J$ Endod. 2002 Apr; 28(4):324-7.

[35]. Nimet Gencoglu, Dilek Helvacioglu; Comparison of the Different Techniques to Remove Fractured Endodontic Instruments from Root Canal Systems; Eur J Dent. 2009 April; 3(2): 90-95.

[36]. Clifford J. Ruddle; Microendodontic NonsurgicaL Retreatment: Silver Point Removal; Dentistry Today February 1997.

[37]. Rubinstein R. A., Kim S. (2002): Long-term followup of cases considered healed one year after apical microsurgery. J Endod 2002; 28: 378-383.

[38]. Tsesis I., Rosen E., Schwartz-Arad D., Fuss Z. (2006): Retrospective Evaluation of Surgical Endodontic Treatment: Traditional versus Modern Technique. J Endod 2006; 32(5): 412-416.

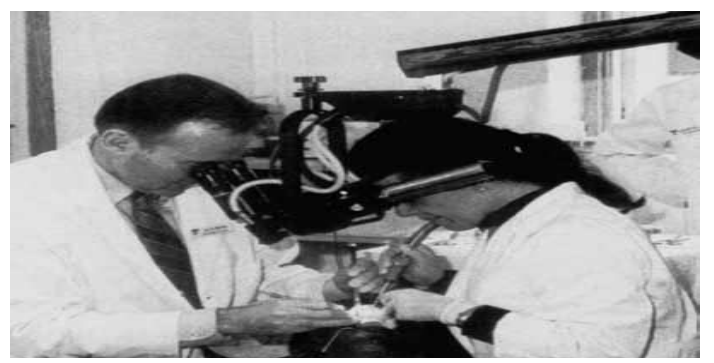

Fig 1.The Dentiscope - the first DOM — in use.

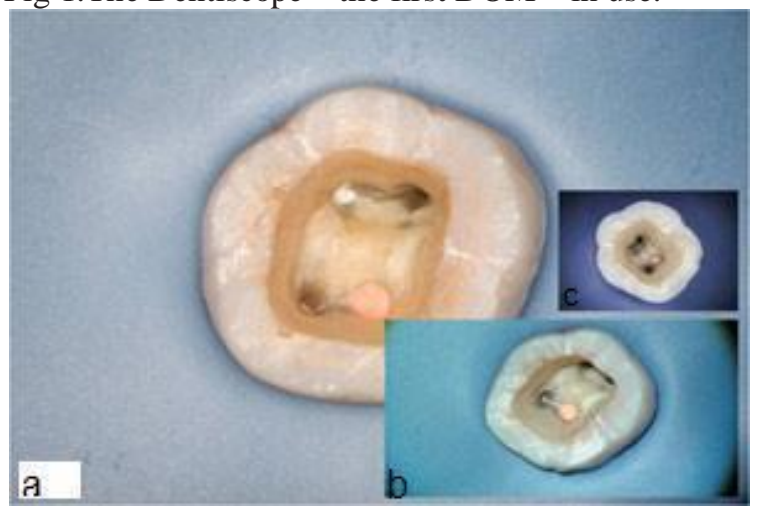

Fig.3: Comparision of magnification between DOM (a), loupes (b) and naked eye(c)

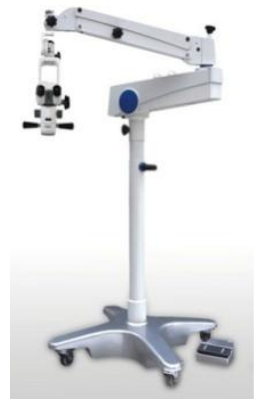

Fig.2: Modern Dental Operating Microscope

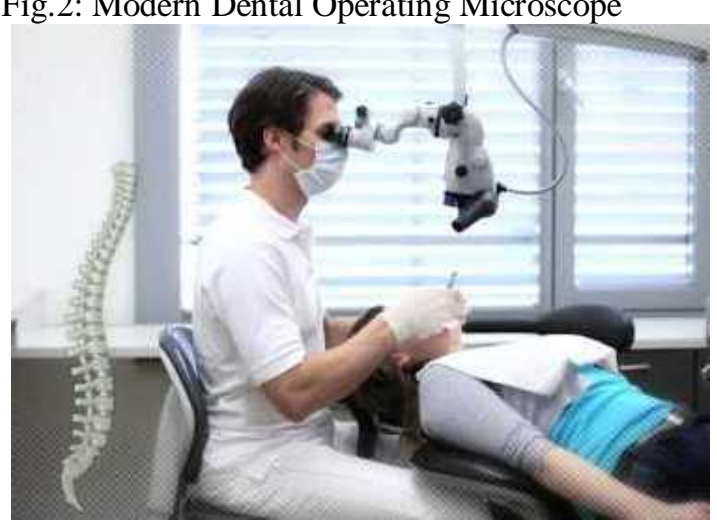

Fig.4: Improved ergonomics during working with dental operating microscope 


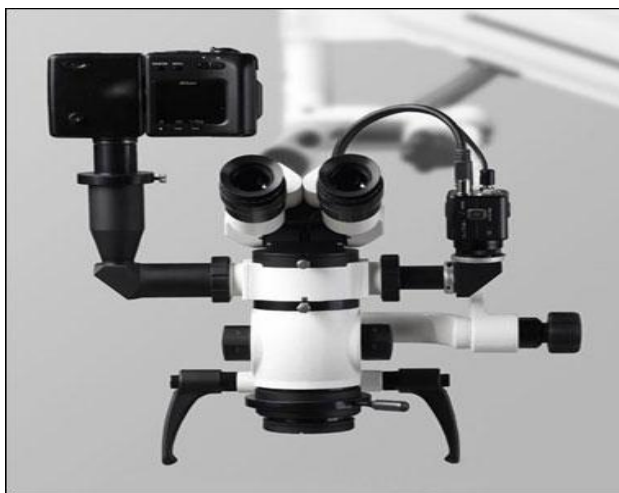

Fig.5: Microscope attached with digital camera for proper documentation

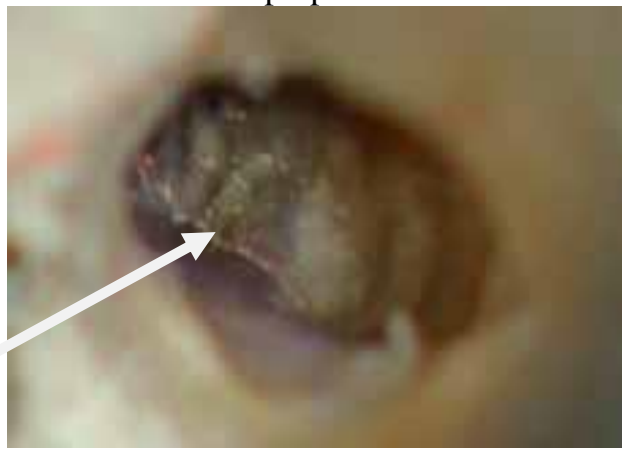

Fig. 7: Microfracture diagnosed during orthograde root canal treatment

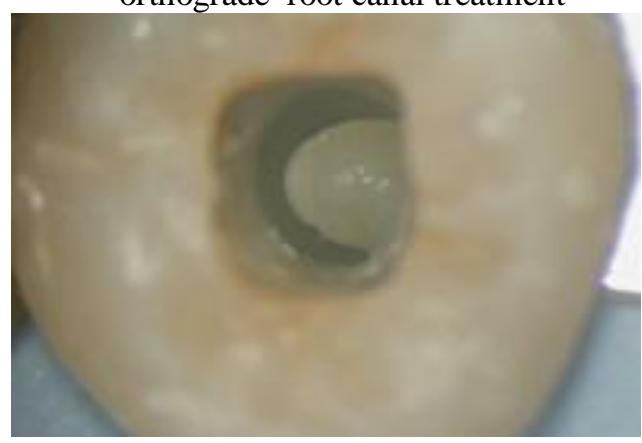

Fig. 9: Excavation of a C-shaped root canal

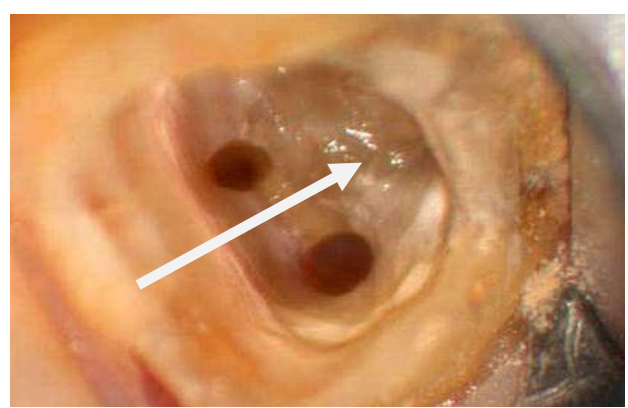

Fig. 11: Obliterated canal orifices impair instrumentation or even prevent root canal treatment

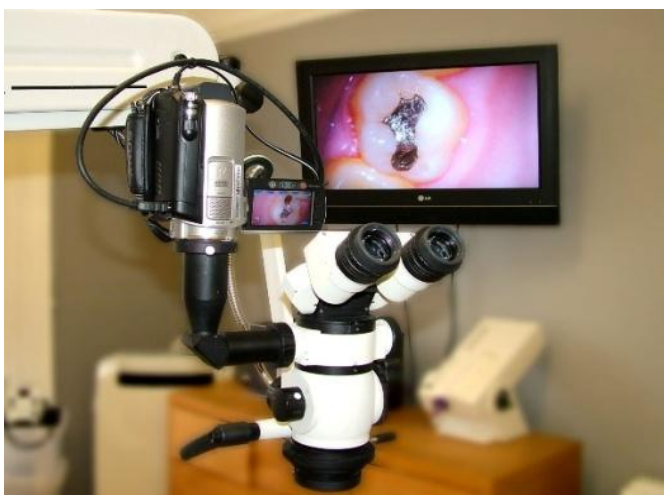

Fig.6: Microscope attached with digital SLR or video camera for communication

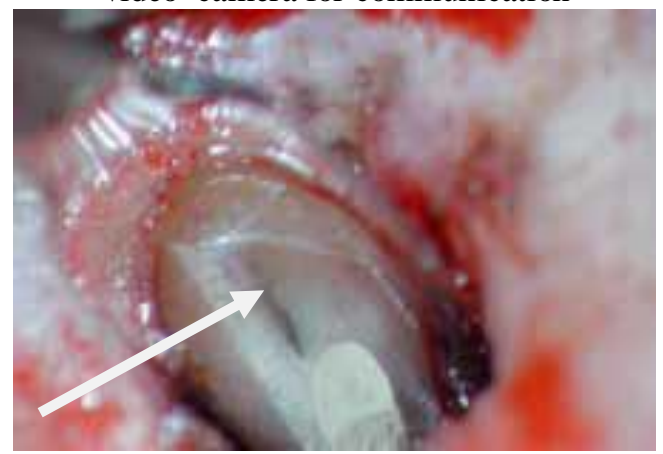

Fig. 8: Microfracture diagnosed during microsurgical endodontic treatment

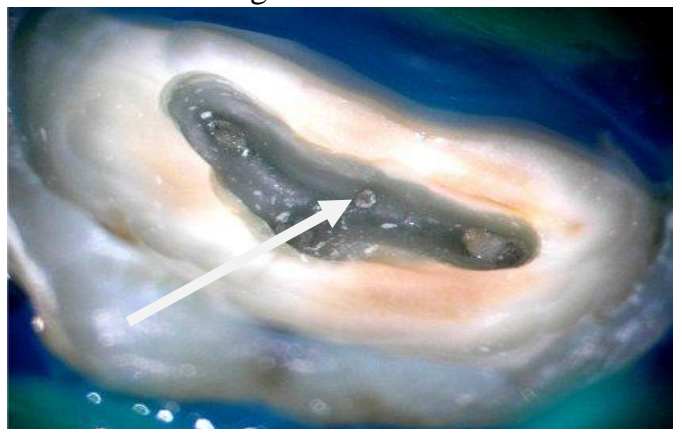

Fig. 10: Localization of the second mesiobuccal canal (MB II) of an upper first molar

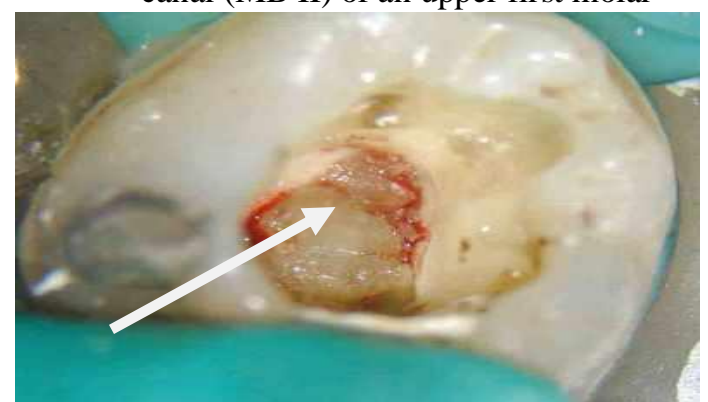

Fig. 12: Denticles may block the canal entrance 


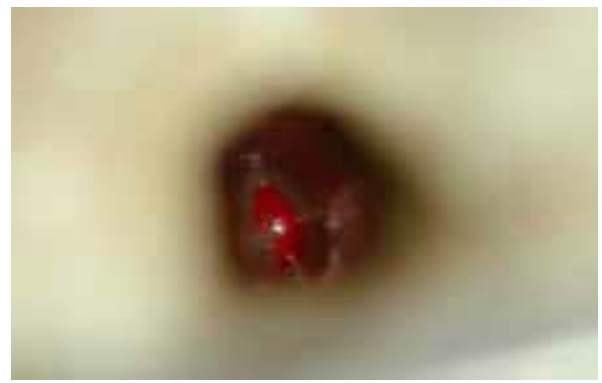

Fig.13.Localization of the root canal end

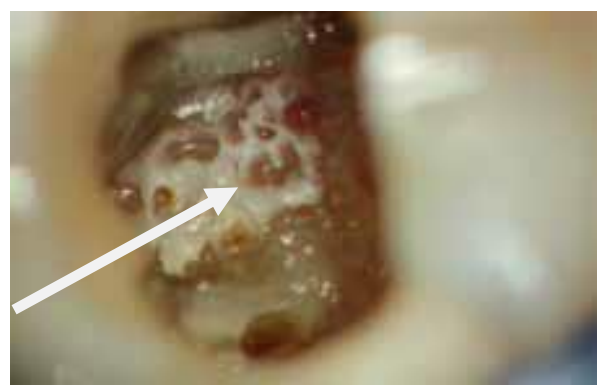

Fig. 15: Cleaned out perforation site and visible bone in the furcation

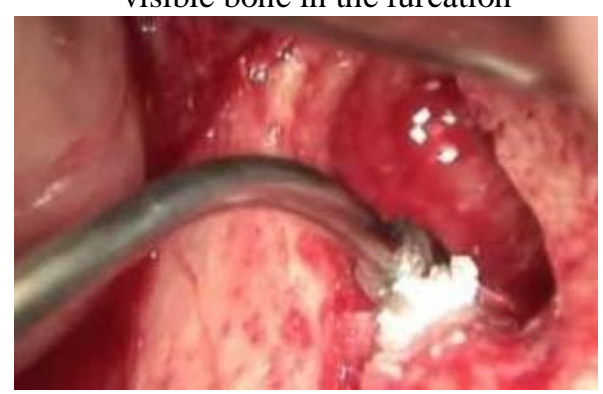

Fig. 17: Microsurgical retro preparation and retrofill with MTA

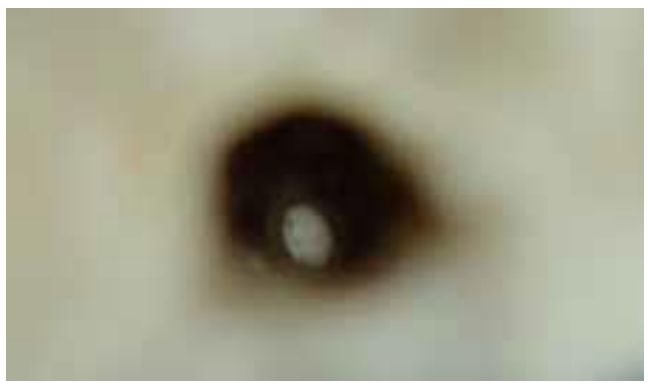

Fig. 14: Creation of a barrier across the open open apex, before obturation

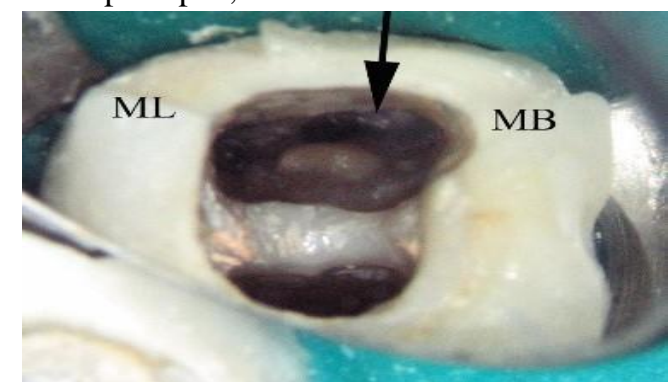

Fig. 16: Visualization of a fractured instrument is essential for retrieval 